\title{
Scale sizes of magnetosheath jets
}

\author{
F. Plaschke ${ }^{1}$, H. Hietala ${ }^{2}$, and Z. Vörös ${ }^{1}$ \\ ${ }^{1}$ Space Research Institute, Austrian Academy of Sciences. \\ ${ }^{2}$ Department of Physics, Imperial College London. \\ ${ }^{2}$ Blackett Laboratory, Prince Consort Road, London SW7 2AZ, United Kingdom.
}

\section{$7 \quad$ Key Points:}

- We report the first unbiased distributions of scale sizes of magnetosheath jets

- Most magnetosheath jets are an order of magnitude smaller than previously reported

- Hundreds to thousands of small scale jets potentially impact the dayside magnetopause every hour

Corresponding author: Ferdinand Plaschke, ferdinand.plaschke@oeaw.ac.at 


\begin{abstract}
Magnetosheath jets are plasma entities that feature a significantly enhanced dynamic pressure with respect to the ambient plasma. They occur more often downstream of the quasi-parallel bow shock. Jets can propagate through the entire magnetosheath and impact on the magnetopause. We reanalyze multi-spacecraft data from the Time History of Events and Macroscale Interactions during Substorms (THEMIS) mission to obtain the first unbiased distributions of scale sizes of the jets, in the directions parallel and perpendicular to their propagation direction. These distributions are log-normal; they fit well to the observations. We argue that jet scales should be log-normally distributed as they should result from multiplicative processes in the foreshock and in the magnetosheath. We find that typical jet scales are on the order of 0.1 Earth radii $\left(R_{\mathrm{E}}\right)$, one order of magnitude smaller than previously reported. Median scale sizes of $0.12 R_{\mathrm{E}}$ and $0.15 R_{\mathrm{E}}$ in the perpendicular and parallel directions are obtained. The small scales may be related to the substructure of Short Large Amplitude Magnetic Structures (SLAMS) in the foreshock, or to the break up of larger jets within the magnetosheath. Use of the log-normal distributions also allows for an analysis of impact rates of small scale jets: While previous results on large jets hitting the magnetopause several times per hour remain largely unchanged, we now find that hundreds to thousands of mostly small scale jets could potentially impact the dayside magnetopause every hour.
\end{abstract}

\title{
1 Introduction
}

The subsolar magnetosheath is often permeated by high-speed jets. These are localized plasma entities featuring significantly enhanced dynamic pressure with respect to their environment (for a recent review, see Plaschke et al., 2018). Jets may be generated at shock ripples or undulations that stem from impinging foreshock structures merging with the shock (Hietala et al., 2009, 2012; Hietala \& Plaschke, 2013; Karlsson et al., 2015; Hao et al., 2016; Palmroth et al., 2018). Consequently, jets are more frequently observed in the subsolar magnetosheath when the cone angle of the interplanetary magnetic field (IMF) with the Sun-Earth-line is small (Archer \& Horbury, 2013; Plaschke et al., 2013; Vuorinen et al., 2019), i.e., downstream of the quasi-parallel bow shock. Notwithstanding, a sizable minority of jets are suggested to be associated with discontinuities in the IMF rather than with low IMF cone angle conditions (e.g., Archer et al., 2012; Savin et al., 2012; Plaschke et al., 2017).

Jets can penetrate the magnetosheath all the way to the magnetopause, where they are known to generate severe, local boundary indentations on impact (e.g., Shue et al., 2009; Amata et al., 2011). As a consequence, (standing) magnetopause surface waves may be launched (e.g., Plaschke \& Glassmeier, 2011; Archer et al., 2019) and magnetopause reconnection may be triggered or modulated at the impact site (Hietala et al., 2018). The consequences of jet impacts can be observed inside the magnetosphere and even from the ground, as they can cause geomagnetic field variations, ionospheric flow enhancements, and dayside auroral features, e.g., local diffuse aurora brightenings (Hietala et al., 2012; Han et al., 2017; Han et al., 2018, 2019; Wang et al., 2018). Hence, jets constitute a coupling element between processes in the foreshock and at the bow shock with consequences at the magnetopause, in the magnetosphere, in the ionosphere, and on the ground.

The importance of jets is related to how much mass, momentum, and energy they transfer from the shock to the magnetopause. Larger jets will more likely have larger effects than smaller jets, but if those jets were rare, then their overall magnetospheric effect would be limited. However, Plaschke et al. (2016) found that large scale jets hit the dayside magnetopause very frequently: The impact rate of jets with cross-sectional diameters over $2 R_{\mathrm{E}}$ ( 2 Earth radii) is approximately 3 per hour, in general. Under low IMF cone angle conditions $\left(<30^{\circ}\right)$, this rate surpasses 9 impacts per hour. 


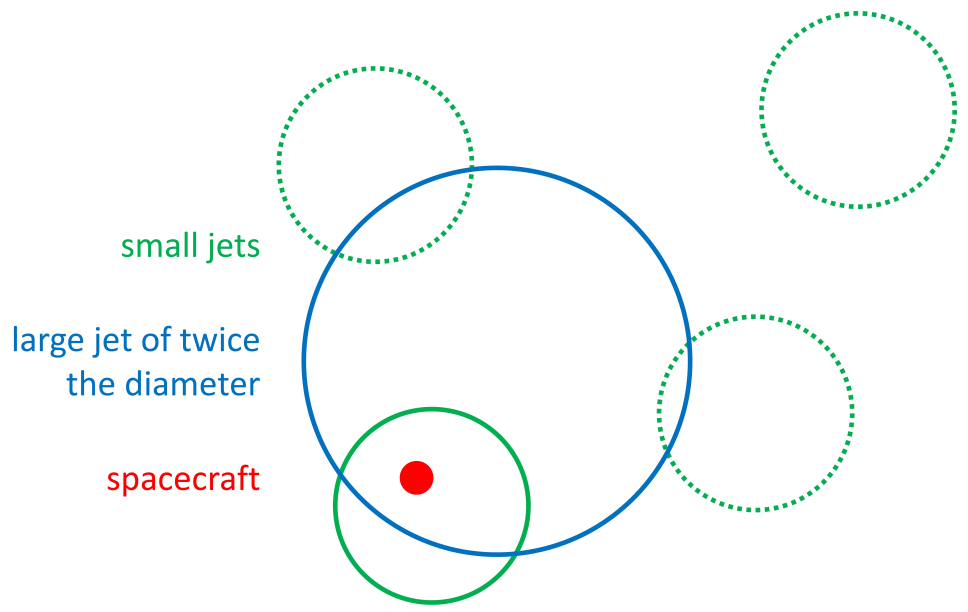

Figure 1. The large jet (blue) has twice the diameter $D_{\perp}$ of the small jets (green). The spacecraft (red) is, hence, four times more likely to cross and observe the large jet than any of the small jets. In this case, three small jets (green dotted lines) are missed.

As an intermediate step towards this result, Plaschke et al. (2016) obtained distributions of observed jet scale sizes, based on Time History of Events and Macroscale Interactions during Substorms (THEMIS, Angelopoulos, 2008) multi-spacecraft measurements of jets in the years 2008 to 2011. These distributions have the following form:

$$
P_{\exp }(D)=\frac{1}{D_{0}} \exp \left(-\frac{D}{D_{0}}\right)
$$

Here, $D$ denotes the jet diameter parallel or perpendicular to its propagation direction ( $D_{\|}$or $D_{\perp}$, respectively). A circular cross-section in the perpendicular plane was assumed by Plaschke et al. (2016). They found the characteristic scale sizes to be $D_{\| 0}=0.71 R_{\mathrm{E}}$ and $D_{\perp 0}=1.34 R_{\mathrm{E}}$. This is in line with previous studies reporting jet scale sizes close to $1 R_{\mathrm{E}}$ (e.g., Němeček et al., 1998; Savin et al., 2012; Archer \& Horbury, 2013; Plaschke et al., 2013). Larger upper limit scale size estimates of a few $R_{\mathrm{E}}$ were found by Hietala et al. (2012) and Gunell et al. (2014), while smaller scales between 0.2 and $0.5 R_{\mathrm{E}}$ in the perpendicular direction have been reported by Archer et al. (2012) for jets generated by IMF discontinuities.

It is crucial to note that the distributions $P_{\exp , \|}$ and $P_{\exp , \perp}$ pertain only to the sample of observed jets; they do not reflect the "true" distributions of scale sizes over all jets in the subsolar magnetosheath. The reason for this discrepancy is the observational bias towards jets with larger perpendicular, i.e., cross-sectional scales. The probability of observation of jets by (single) spacecraft is proportional to $D_{\perp}^{2}$ (see Figure 1). This fact has been taken into account by Plaschke et al. (2016) when computing the impact rates: In the appropriate integral, a correction factor $\sim 1 / D_{\perp}^{2}$ is applied to $P_{\exp , \perp}$. Unfortunately, this trick does not help when seeking unbiased distributions of scale sizes, as $P_{\exp } / D^{2}$ cannot be normalized:

$$
\int_{0}^{\infty} \frac{1}{D_{0} D^{2}} \exp \left(-\frac{D}{D_{0}}\right) \mathrm{d} D=\infty
$$

Here, the ansatz (1) is the root cause, as $P_{\exp }$ does not vanish at $D=0$. It vastly overestimates the probability to observe smallest jets: $P_{\exp }$ even maximizes at $D=0$ while naturally there should be a physical lower limit on the jet size. 
In this paper, we reanalyze the observations of Plaschke et al. (2016) assuming a more appropriate log-normal distribution of the form:

$$
P_{\ln }(D)=\frac{1}{D \sigma \sqrt{2 \pi}} \exp \left(-\frac{\left(\ln \left(D / R_{\mathrm{E}}\right)-\mu\right)^{2}}{2 \sigma^{2}}\right)
$$

as a model for the unbiased scale size distributions. Here $\mu$ and $\sigma$ are unitless and $D$ is given in $R_{\mathrm{E}}$. The parameters $\mu$ and $\sigma$ represent the mean and the standard deviation of the natural logarithm of the scale sizes $D$. Equation (3) has to be multiplied with $D^{2}$ to reflect the distributions of observed jet scale sizes. After normalization, we obtain the following modified log-normal distribution:

$$
P_{\text {mod }}(D)=\frac{D / R_{\mathrm{E}}^{2}}{\sigma \sqrt{2 \pi}} \exp \left(-2 \mu-2 \sigma^{2}-\frac{\left(\ln \left(D / R_{\mathrm{E}}\right)-\mu\right)^{2}}{2 \sigma^{2}}\right)
$$

We use this equation as a substitute for Equation (1). With its help, we are able to determine $\sigma$ and $\mu$ and, therefore, to obtain, for the first time, corrected, unbiased jet scale size distributions (Equation 3). These distributions are currently in demand as standards of comparison for simulations focusing on magnetosheath jets (e.g., Hao et al., 2016; Palmroth et al., 2018).

Note that on computing $P_{\text {mod, } \|}$, we multiply $P_{\mathrm{ln}, \|}$ with $D_{\|}^{2}$ before renormalization. However, the actual correction factor should be $D_{\perp}^{2}$, because the probability of jet observation scales with $D_{\perp}^{2}$ (see Figure 1). Here we implicitly assume that jets keep their aspect ratio, e.g., that jets of large/small $D_{\perp}$ are also large/small in $D_{\|}$. If we assume, instead, that $D_{\|}$and $D_{\perp}$ are independent to each other, then we should fit the observed distribution of parallel scale sizes directly with $P_{\mathrm{ln}, \|}$ (Equation 3). As we show in section 6 , there are indications in the data that the truth is in between these two assumptions.

\section{Justification of the Log-Normal Distribution}

The assumption that the log-normal distribution is a good model for the jet scale sizes should be justified. To achieve this, we recall some of the basic properties of the normal and log-normal distributions: The normal distribution can be interpreted to result from additive processes. The central limit theorem states that the sums $Y$ of $n$ independent and identically distributed variables $X$, i.e., $Y=X_{1}+\ldots+X_{n}$, tend to be normally distributed as $n \rightarrow \infty$. The exponential functions $Z$ of the normally distributed variables $Y$, i.e., $Z=\exp (Y)$, will in turn be $\log$-normally distributed. $Z$ can also be written as the product of the variables $\exp \left(X_{1}\right) \ldots \exp \left(X_{n}\right)$; in fact, the products $Z$ of any $n$ independent and identically distributed variables $X$, i.e., $Z=X_{1} \ldots X_{n}$, tend to be log-normally distributed as $n \rightarrow \infty$. Hence, the log-normal distribution can be interpreted to result from multiplicative processes (e.g., Vörös et al., 2015).

We follow the argumentation outlined in section 2 of Campbell (1995) to show that it is reasonable to assume that the jet scales result from multiplicative processes: Let us assume that magnetosheath jets start at the bow shock with scale sizes $D_{\text {init }}$, and that their scales $D$ change randomly over time $t$ as jets propagate through the magnetosheath:

$$
\frac{\mathrm{d} D}{\mathrm{~d} t}=K(t) D(t)
$$

Here $K(t)$ is the growth rate applied at time $t$, which can be assumed to randomly fluctuate. The solution to Equation (5) is:

$$
D(t)=D_{\text {init }} \exp \left(\int_{0}^{t} K(t) \mathrm{d} t\right)
$$


Taking the logarithm of this equation and expressing the integral as a sum of $n$ integrals over shorter time intervals yields (equation 3 in Campbell, 1995):

$$
\ln (D(t))=\ln \left(D_{\text {init }}\right)+\sum_{i=1}^{n} \int_{t_{i-1}}^{t_{i}} K(t) \mathrm{d} t
$$

As $K$ is a random variable, so are each of the integrals. Hence, for large $n$, the right term represents a sum of independent and identically distributed variables: it should be normally distributed and the exponential function of that term (see Equation 6) should be log-normally distributed. The product of two log-normally distributed variables is itself log-normally distributed. Hence, $D$ should be log-normally distributed if this condition holds for $D_{\text {init }}$. In the discussion section 8 , we argue that this should be true by repeating the above line of arguments.

\section{Data Sets}

The jet data sets used in this study are identical to the ones used in Plaschke et al. (2016), based on earlier work by Plaschke et al. (2013). We briefly recall in this section how they have been obtained. This section, hence, briefly summarizes sections 2 and 3 of Plaschke et al. (2016).

The underlying measurements have been performed by the five THEMIS spacecraft (Angelopoulos, 2008) between 2008 and 2011 (see section 2 in Plaschke et al., 2013): First, orbit segments are preselected inside a $30^{\circ}$ wide cone with the tip at Earth and open towards the Sun, with spacecraft distances to Earth ranging from 7 to $18 R_{\mathrm{E}}$. Therein, magnetosheath data intervals are mainly selected by comparison of THEMIS density measurements by the Electrostatic Analyzers (ESA, McFadden et al., 2008) with upstream solar wind measurements, as given by NASA's OMNI data set (King \& Papitashvili, 2005). The magnetosheath density is required to exceed twice the corresponding solar wind value. In addition, ESA ion energy flux measurements at $10 \mathrm{keV}$ are required to be lower than at $1 \mathrm{keV}$, to exclude magnetospheric observations. Magnetosheath intervals should exceed 2 minutes in length, and magnetic field and ion moments from THEMIS and OMNI should be available throughout. Note that OMNI solar wind measurements are averaged over intervals of 5 minutes preceding times of comparison to THEMIS data, to account for delays due to the flow of plasma from the bow shock to the THEMIS spacecraft locations inside the magnetosheath.

The above criteria yield a total of 2736.9 hours of magnetosheath measurements by all five THEMIS spacecraft spread over 6960 individual intervals. The times of these intervals can be found in Plaschke, Hietala, and Angelopoulos (2020). Within these intervals, jets are mainly identified by the dynamic pressure in anti-sunward direction surpassing half the corresponding value in the solar wind $\left(p_{\mathrm{d}, x}=\rho v_{x}^{2}>p_{\mathrm{d}, \mathrm{sw}} / 2\right)$. Here, $x$ refers to the sunward component in the geocentric solar ecliptic (GSE) coordinate system; the time of maximum dynamic pressure ratio $p_{\mathrm{d}, x} / p_{\mathrm{d}, \mathrm{sw}}$ is denoted with $t_{0}$. If this criterion is fulfilled, then jet intervals of length $t_{\text {jet }}$ are defined by $p_{\mathrm{d}, x}>p_{\mathrm{d}, \mathrm{sw}} / 4$. Note that $p_{\mathrm{d}, x}=p_{\mathrm{d}, \mathrm{sw}} / 4$ is the maximum dynamic pressure possible just downstream of the smooth, subsolar shock in the high Alfvén Mach number limit. The jet identification limit is set to twice that threshold to avoid false detections of random fluctuations in dynamic pressure instead of coherent jet structures. The following additional criteria help in achieving this goal: Within jet intervals, $v_{x}<0$ should hold. In both 1-minute intervals before and after the jet intervals, the pre- and post-jet intervals, $v_{x}>v_{x}\left(t_{0}\right) / 2$ should be fulfilled at least once. In addition, all pre-jet, jet, and post-jet intervals should lie within one magnetosheath interval as defined above. With these criteria, 2859 jets are identified (Plaschke et al., 2013; Plaschke, Hietala, \& Angelopoulos, 2020). Out of these, the majority of 2126 jets has been observed by the inner THEMIS spacecraft THA, THD, and THE closer to the magnetopause than to the bow shock. Note that short-period so- 


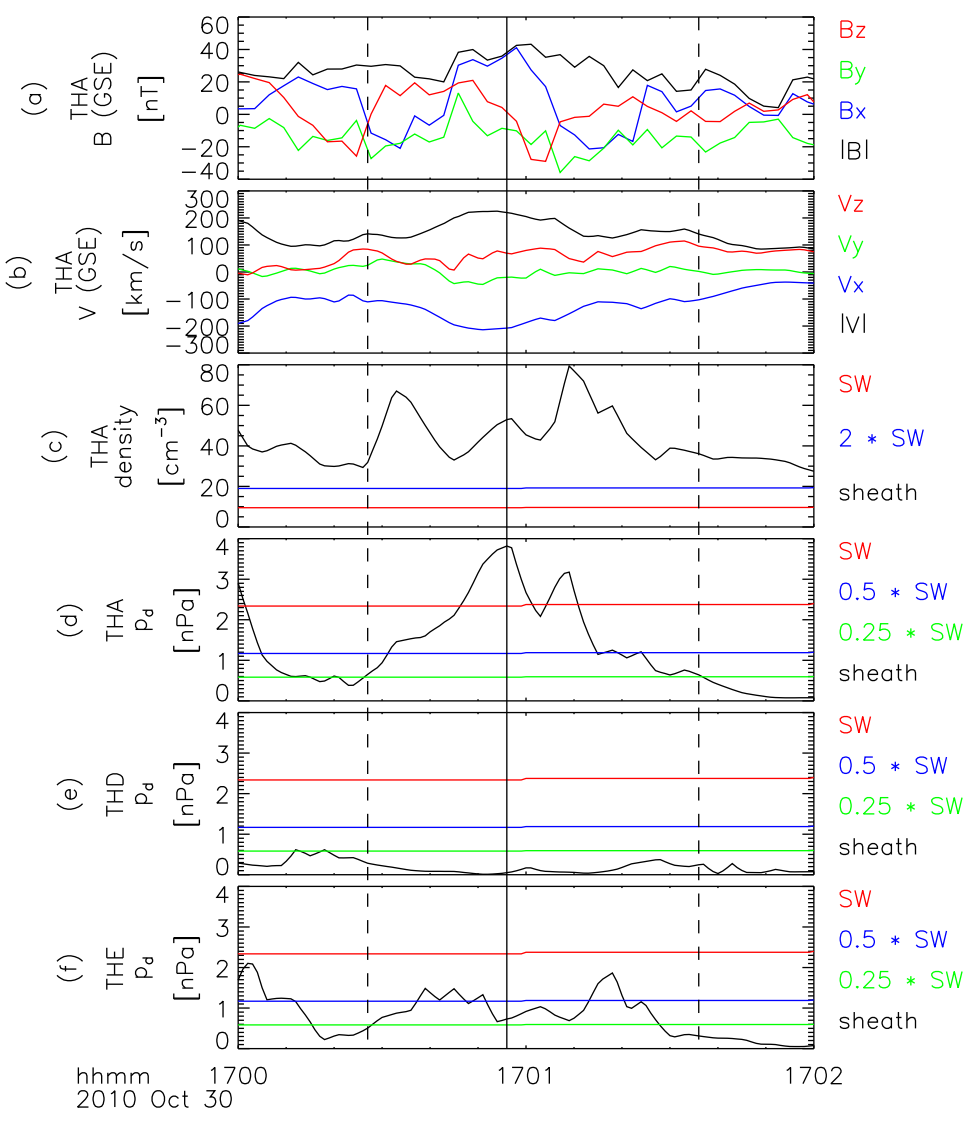

Figure 2. Example of a jet interval: observations by THA, THD, and THE in the magnetosheath and from the OMNI data set in the solar wind. From top to bottom: (a and b) THA magnetic field and ion velocity measurements in GSE; (c) THA ion density measurements in black and (twice the) solar wind ion density in red (blue); (d to f) THA, THD, and THE dynamic pressure $\left(p_{\mathrm{d}, x}\right)$ observations in black and corresponding solar wind data in red, blue, and green, showing $p_{\mathrm{d}, \mathrm{sw}}, p_{\mathrm{d}, \mathrm{sw}} / 2$ and $p_{\mathrm{d}, \mathrm{sw}} / 4$, respectively. Vertical dotted lines delimit the jet interval of a jet observed by THA (see panel c) and the solid vertical line shows the corresponding time of maximum dynamic pressure ratio $t_{0}$.

lar wind fluctuations cannot contribute to the identification of jets, due to the 5-minute averaging applied to the OMNI solar wind data.

For all jets and corresponding jet-observing reference spacecraft, we check if a second THEMIS spacecraft is in the vicinity providing additional context for the observations. In detail it is checked, if the vector $\vec{d}$ from the reference to the second spacecraft and the vector $\vec{v}\left(t_{0}\right)$ form an angle between $80^{\circ}$ and $100^{\circ}$ at time $t_{0}$. Here, $\vec{v}\left(t_{0}\right)$ denotes the ion velocity measured by the reference spacecraft. In addition, we require the second spacecraft to be located in the magnetosheath within $t_{\text {jet }}+1$ min of $t_{0}$. These criteria are fulfilled in 662 cases. In these cases, a second spacecraft is positioned roughly in a plane perpendicular to the jet propagation direction, together with the jet-observing reference spacecraft. Plaschke et al. (2016) denote this sample of cases as two spacecraft (2SC) data set. This data set contains almost only combinations of the inner THEMIS spacecraft THA, THD, and THE. The data set can be retrieved from Plaschke, Hietala, Angelopoulos, and Nakamura (2020). 
Table 1. Probabilities $P_{\mathrm{s}}$ to observe jets simultaneously by reference and second spacecraft, and underlying data ${ }^{a}$

\begin{tabular}{cccccc}
\hline $\begin{array}{c}\text { Bin } \\
i\end{array}$ & $\begin{array}{c}d \text { Range } \\
\left(R_{\mathrm{E}}\right)\end{array}$ & $\begin{array}{c}\text { Average } d \\
\bar{d}\left(R_{\mathrm{E}}\right)\end{array}$ & $\begin{array}{c}\text { \# Cases } \\
N\end{array}$ & $\begin{array}{c}\text { \# Sec. Obs. } \\
N_{\mathrm{s}}\end{array}$ & $\begin{array}{c}P_{\mathrm{s}} \\
(\%)\end{array}$ \\
\hline 1 & $0.0-0.3$ & 0.20 & 111 & 70 & $63 \%$ \\
2 & $0.3-0.6$ & 0.45 & 270 & 102 & $38 \%$ \\
3 & $0.6-0.9$ & 0.64 & 159 & 44 & $28 \%$ \\
4 & $0.9-1.2$ & 1.08 & 58 & 12 & $21 \%$ \\
5 & $1.2-1.7$ & 1.40 & 22 & 1 & $5 \%$ \\
6 & $1.7-2.2$ & 1.94 & 16 & 0 & $0 \%$ \\
7 & $2.2-2.7$ & 2.45 & 13 & 1 & $8 \%$ \\
8 & $2.7-3.2$ & 2.96 & 5 & 0 & $0 \%$ \\
\hline
\end{tabular}

${ }^{a}$ Reproduced from Table 2 in Plaschke et al. (2016).

Example jet observations by THA on 30 October 2010 are shown in Figure 2. Panels (a) to (d) of this figure show the magnetic field, the ion velocity, the ion density, and the corresponding dynamic pressure $\left(p_{\mathrm{d}, x}\right)$ measurements. A jet is identified between 17:00:27 and 17:01:36 UT; dotted vertical lines delimit the jet interval, where $p_{\mathrm{d}, x}>p_{\mathrm{d}, \mathrm{sw}} / 4$ holds. The maximum dynamic pressure ratio $p_{\mathrm{d}, x} / p_{\mathrm{d}, \mathrm{sw}}$ was reached at 17:00:56 UT $\left(t_{0}\right.$, vertical solid line in the figure). At this time, both THD and THE were found to fulfill the 2SC data set criteria stated in the previous paragraph. They provide additional context for the observations of THA. As can be seen in the bottom panels (e) and (f) of Figure 2 , THE also observed the jet at roughly the same time while THD did not. This is related to the inter-spacecraft distances $d$ : Between THA and THD, $d$ was $0.63 R_{\mathrm{E}}$, while it was just $0.17 R_{\mathrm{E}}$ between THA and THE.

\section{Perpendicular Scale Sizes}

The 2SC data set enables us to infer the distribution of observed and, ultimately, the corrected and unbiased jet scale sizes $D_{\perp}$ in the plane perpendicular to the jet propagation direction. The reason is that the probability $P_{\mathrm{s}}$ of observing a jet simultaneously at the reference and second spacecraft as a function of $d=|\vec{d}|$ changes with that distribution. As described in section 4 of Plaschke et al. (2016), we can obtain $P_{\mathrm{s}}(d)$ from the 2SC data: We group the 662 cases into 8 bins with respect to $d$. The bin boundaries are at $d=0.0,0.3,0.6,0.9,1.2,1.7,2.2,2.7$, and $3.2 R_{\mathrm{E}}$ (see Table 1 which reproduces Table 2 in Plaschke et al., 2016). For each bin, we assess the total number of cases $N$ and the subset thereof $N_{\mathrm{s}}$ where the second spacecraft observed a jet simultaneously with the reference spacecraft. This is fulfilled if $t_{0}$, as observed by the reference spacecraft, is within a jet interval $t_{\text {jet }}$, as observed by the second spacecraft. The numbers $N, N_{\mathrm{s}}$, and $P_{\mathrm{s}}=N_{\mathrm{s}} / N$, as well as the average distances $\bar{d}$ in each bin are stated in Table 1 . $P_{\mathrm{s}}(\bar{d})$ is also shown by black crosses in Figure 3 .

Assuming jets have a circular cross section of a single diameter $D_{\perp}$ in the jet-perpendicular plane, then $P_{\mathrm{s}}$ could be reproduced by the following function (see Equation 2 and appendix A in Plaschke et al., 2016):

$$
P_{\mathrm{s}, 1}=\left\{\begin{aligned}
\frac{2}{\pi} \arccos \left(\frac{d}{D_{\perp}}\right)-\frac{2 d}{\pi D_{\perp}^{2}} \sqrt{D_{\perp}^{2}-d^{2}}, & \text { for } d<D_{\perp} \\
0, & \text { for } d \geq D_{\perp}
\end{aligned}\right.
$$

For spacecraft separations larger than that single diameter $\left(d>D_{\perp}\right)$ the second spacecraft would not be able to see the same jet simultaneously. As shown in Plaschke et al. (2016), $P_{\mathrm{s}, 1}$ alone is not a good model for $P_{\mathrm{s}}$. 


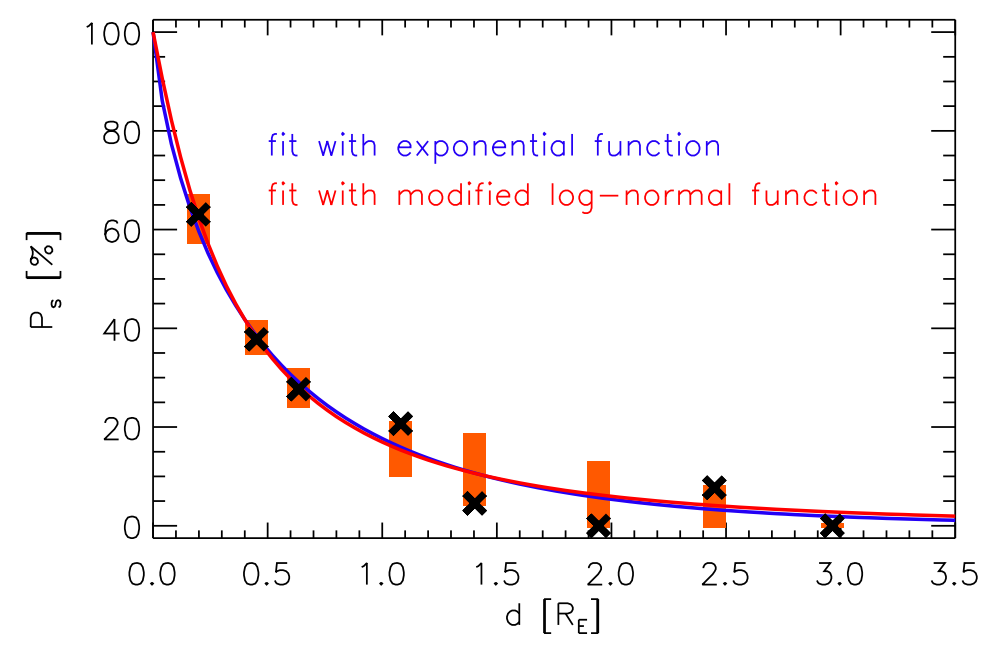

Figure 3. Black crosses show $P_{\mathrm{s}}(\bar{d})$. Blue and red lines show $P_{\mathrm{s}, \mathrm{m}}(d)$ using the exponential and the modified log-normal distributions to characterize the scale sizes of observed jets, respectively. $P_{\mathrm{s}}(\bar{d})$ values should lie within red bars with a probability of $68.2 \%$, as of $P_{\mathrm{s}, \mathrm{m}}(\bar{d})$ model values based on the modified log-normal distribution.

Instead, Plaschke et al. (2016) assume an exponential distribution $P_{\exp , \perp}$ of the form (1) for the observed jet perpendicular scale sizes. Therewith, $P_{\mathrm{s}}$ can be modeled by solving the following integral (Equation 5 in Plaschke et al., 2016):

$$
P_{\mathrm{s}, \mathrm{m}}(d)=\int_{d}^{\infty} P_{\mathrm{s}, 1}\left(d, D_{\perp}\right) P_{\exp , \perp}\left(D_{\perp}\right) \mathrm{d} D_{\perp}
$$

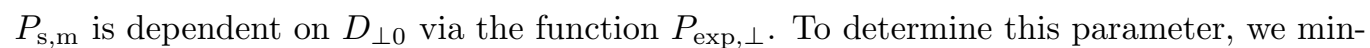
imize the squared difference of $P_{\mathrm{s}, \mathrm{m}}$ and $P_{\mathrm{s}}$, weighted by the numbers of cases $N$ pertaining to each distance bin, as follows (Equation 6 in Plaschke et al., 2016):

$$
\sum_{i}\left(\left(P_{\mathrm{s}, \mathrm{m}}\left(\bar{d}_{i}\right)-P_{\mathrm{s}}\left(\bar{d}_{i}\right)\right)^{2} N_{i}\right)=\min
$$

Here, $i$ is the index of the bin (see first column of Table 1). As discussed in Plaschke et al. (2016), the result is $D_{\perp 0}=1.34 R_{\mathrm{E}}$, and the corresponding $P_{\mathrm{s}, \mathrm{m}}(d)$ is shown in blue in Figure 3.

If we replace $P_{\exp , \perp}$ (Equation 1) by $P_{\bmod , \perp}$ (Equation 4) and redo the minimization, we obtain the parameters pertaining to the log-normal distribution: $\mu_{\perp}=-2.1118$ and $\sigma_{\perp}=0.9991$. The corresponding $P_{\mathrm{s}, \mathrm{m}}(d)$ is shown in red in Figure 3. It approximates the observational $P_{\mathrm{s}}$ values corresponding to lowest distances $d$ (largest numbers of cases $N$ ) slightly better. The red bars in the figure span between the 15.9 and the 84.1 percentiles of the binomial distributions for modified log-normal distribution based $P_{\mathrm{s}, \mathrm{m}}(\bar{d})$ values and numbers $N$. With a probability of $68.2 \%$, the $P_{\mathrm{s}}$ values are expected to lie within the bars. As can be seen in Figure 3, this is always the case, confirming the modified log-normal distribution to appropriately model the observed perpendicular scale sizes of jets.

A larger difference can be observed between the distributions $P_{\exp , \perp}$ and $P_{\bmod , \perp}$. These are shown in Figure 4 by solid black and red lines, respectively. As can be seen, $P_{\text {mod, } \perp}$ drops towards lowest scale sizes $D_{\perp}$, as intended. Conversely, it attributes a larger probability of observation to jets with perpendicular scale sizes between approximately 0.2 and $1.3 R_{\mathrm{E}}$. The median values of the exponential $P_{\exp , \perp}$ and the modified log-normal 


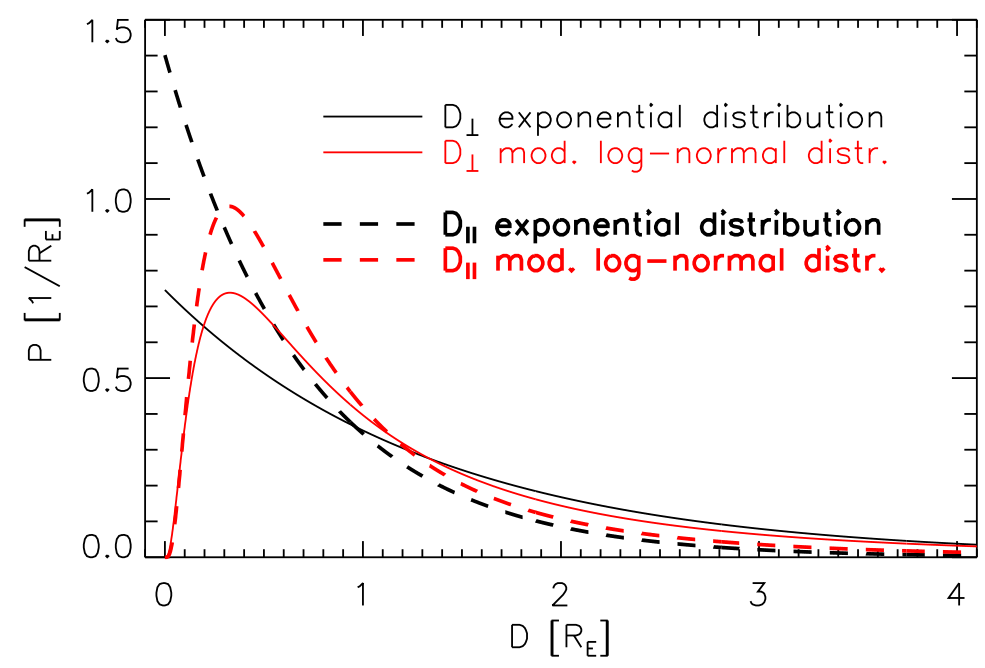

Figure 4. $\quad P_{\perp}$ and $P_{\|}$are shown by solid and dashed lines. The black lines correspond to the exponential distributions $P_{\exp }$ (Equation 1), using the parameters $D_{\perp 0} \quad=\quad 1.34 R_{\mathrm{E}}$ and $D_{\| 0}=0.71 R_{\mathrm{E}}$. The red lines correspond to the modified log-normal distributions $P_{\text {mod }}$ (Equation 4), using the parameters $\mu_{\perp}=-2.1118$ and $\sigma_{\perp}=0.9991$, as well as $\mu_{\|}=-1.8703$ and $\sigma_{\|}=0.8636$, respectively.

$P_{\text {mod }, \perp}$ distributions are given by $\bar{D}_{\exp , \perp}=\ln (2) D_{\perp 0}=0.93 R_{\mathrm{E}}$ and $\bar{D}_{\text {mod }, \perp}=\exp \left(\mu_{\perp}+\right.$ $\left.2 \sigma_{\perp}^{2}\right) R_{\mathrm{E}}=0.89 R_{\mathrm{E}}$; they are very similar. These median values can also be found in Table 2 .

\section{Parallel Scale Sizes}

The observed jet scale sizes in the direction of jet propagation are determined following section 5 of Plaschke et al. (2016). Therefore the subset of 2126 THA, THD, and THE observed jets is used. For every jet, a scale $D_{\|}$can be computed as follows (Equation 7 in Plaschke et al., 2016):

$$
D_{\|}=\int_{t_{\mathrm{jet}}} \frac{\vec{v}\left(t_{0}\right) \cdot \vec{v}(t)}{\left|\vec{v}\left(t_{0}\right)\right|} \mathrm{d} t
$$

The histogram of $D_{\|}$scales is shown in black in Figure 5. Fitting an exponential function of the form $\sim \exp \left(-D_{\|} / D_{\| 0}\right)$ (Equation 1) to this distribution, between $D_{\|}=0.4$ and $8 R_{\mathrm{E}}$, we obtain $D_{\| 0}=0.71 R_{\mathrm{E}}$. The corresponding function is shown in blue in Figure 5 .

Clearly, the decreasing probability at the lower end of $D_{\|}$cannot be reproduced by the exponential function. In this region, the modified log-normal distribution performs much better. A fit with $P_{\text {mod, } \|}$ (Equation 4) yields parameters $\mu_{\|}=-1.8703$ and $\sigma_{\|}=$ 0.8636. The corresponding function is shown in red in Figure 5, which matches the observed distribution over the entire range of $D_{\|}$values.

Figure 4 shows both normalized $P_{\|}$distributions with dashed lines $\left(P_{\text {exp }, \|}\right.$ in black and $P_{\text {mod, } \|}$ in red). $P_{\text {mod, } \|}$ exceeds $P_{\exp , \|}$ for larger $D_{\|}>0.3 R_{\mathrm{E}}$, compensating the drop at smallest scales. The median values of $P_{\text {exp }, \|}$ and $P_{\text {mod, } \|}$ are $\bar{D}_{\text {exp }, \|}=\ln (2) D_{\| 0}=$ $0.49 R_{\mathrm{E}}$ and $\bar{D}_{\mathrm{mod}, \|}=\exp \left(\mu_{\|}+2 \sigma_{\|}^{2}\right) R_{\mathrm{E}}=0.68 R_{\mathrm{E}}$.

Note that using $P_{\ln , \|}$ (Equation 3) for the fit yields an almost identical result with respect to $P_{\text {mod, }, \|}$. In this case, we obtain $\mu_{\|}=-0.3675$ and $\sigma_{\|}=0.8699$, and a me- 


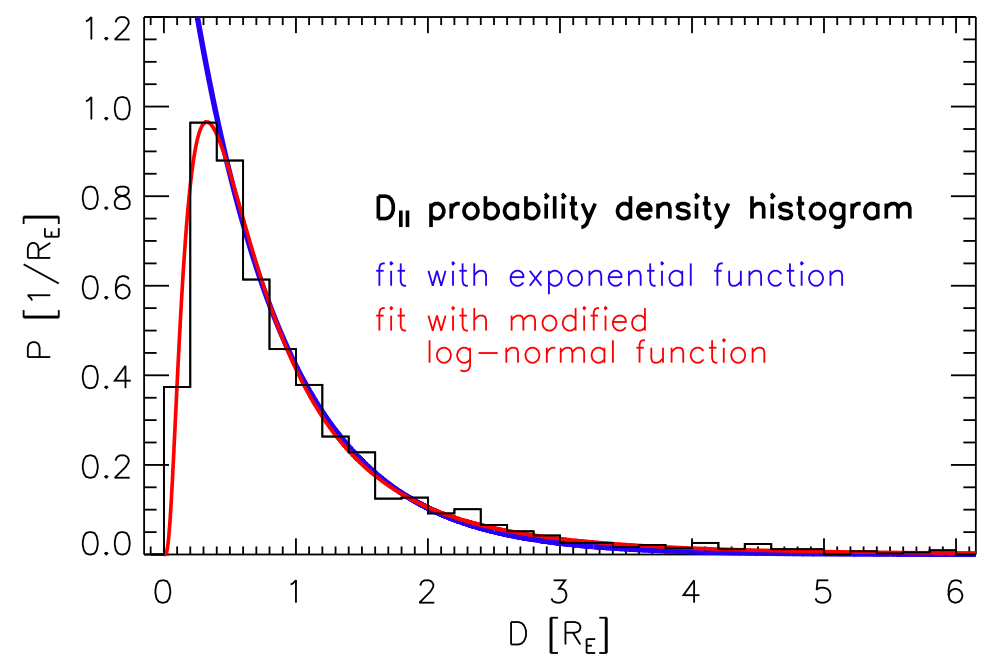

Figure 5. Probability density histogram of observed jet scale sizes $D_{\|}$parallel to their propagation direction is shown in black. Two fitted $P_{\|}$distributions are shown as well: an exponential function according to Equation (1) with $D_{\| 0}=0.71 R_{\mathrm{E}}$ in blue and a modified log-normal distribution according to Equation (4) with $\mu_{\|}=-1.8703$ and $\sigma_{\|}=0.8636$ in red.

Table 2. Median values of jet scale size distributions

\begin{tabular}{cccc}
\hline Direction & $\begin{array}{c}\text { Observed } \\
\bar{D}_{\exp }\left(R_{\mathrm{E}}\right)\end{array}$ & $\begin{array}{c}\text { Observed } \\
\bar{D}_{\text {mod }}\left(R_{\mathrm{E}}\right)\end{array}$ & $\begin{array}{c}\text { Unbiased/Corrected } \\
\bar{D}_{\ln }\left(R_{\mathrm{E}}\right)\end{array}$ \\
\hline$\perp$ & 0.93 & 0.89 & 0.12 \\
$\|$ & 0.49 & 0.68 & 0.15 \\
\hline
\end{tabular}

dian value of $\bar{D}_{\mathrm{ln}, \|}=\exp \left(\mu_{\|}\right) R_{\mathrm{E}}=0.69 R_{\mathrm{E}} \approx \bar{D}_{\text {mod, }, \|}$. Such a fit would be justified if we could assume $D_{\|}$to be independent of $D_{\perp}$. In this case, the fitted distribution $P_{\mathrm{ln}, \|}$ would already represent the unbiased scale size distribution.

In Figure $4, P_{\|}$and $P_{\perp}$ can be directly compared. As $D_{\| 0}$ is approximately half as large as $D_{\perp 0}$, the parallel scale distributions $P_{\text {exp, } \|}$ and also $P_{\text {mod, } \|}$ show larger/smaller probabilities in general for lower/higher scale values $D$ than the perpendicular scale distributions $P_{\exp , \perp}$ and $P_{\bmod , \perp}$. The cross-over point is around $1 R_{\mathrm{E}}$. Interestingly, both modified $\log$-normal distributions $P_{\text {mod, } \|}$ and $P_{\text {mod, } \perp}$ have a similar shape and are almost identical around $D \approx 0.1 R_{\mathrm{E}}$. Below that value, $P_{\text {mod, } \perp}$ even slightly exceeds $P_{\text {mod, } \|}$ - a fact that becomes important below.

\section{Unbiased Scale Size Distributions}

As discussed in the introduction, we can correct the modified log-normal distributions and convert them from observed jet scale size distributions to unbiased jet scale size distributions. Therefore, we have to multiply them with a factor $\sim 1 / D^{2}$ and renormalize them, obtaining by construction log-normal distributions $P_{\ln }$ (Equation 3 ). The same parameters $\mu_{\perp}=-2.1118$ and $\sigma_{\perp}=0.9991$, as well as $\mu_{\|}=-1.8703$ and $\sigma_{\|}=$ 0.8636 , as determined above, apply to the unbiased scale size distributions $P_{\mathrm{ln}, \perp}$ and $P_{\mathrm{ln}, \|}$.

$P_{\ln , \perp}$ and $P_{\ln , \|}$ are shown in Figure 6 by solid and dashed lines, respectively. Apparently, the distributions are very similar, much more so than $P_{\exp , \perp}$ and $P_{\exp , \|}$. This 


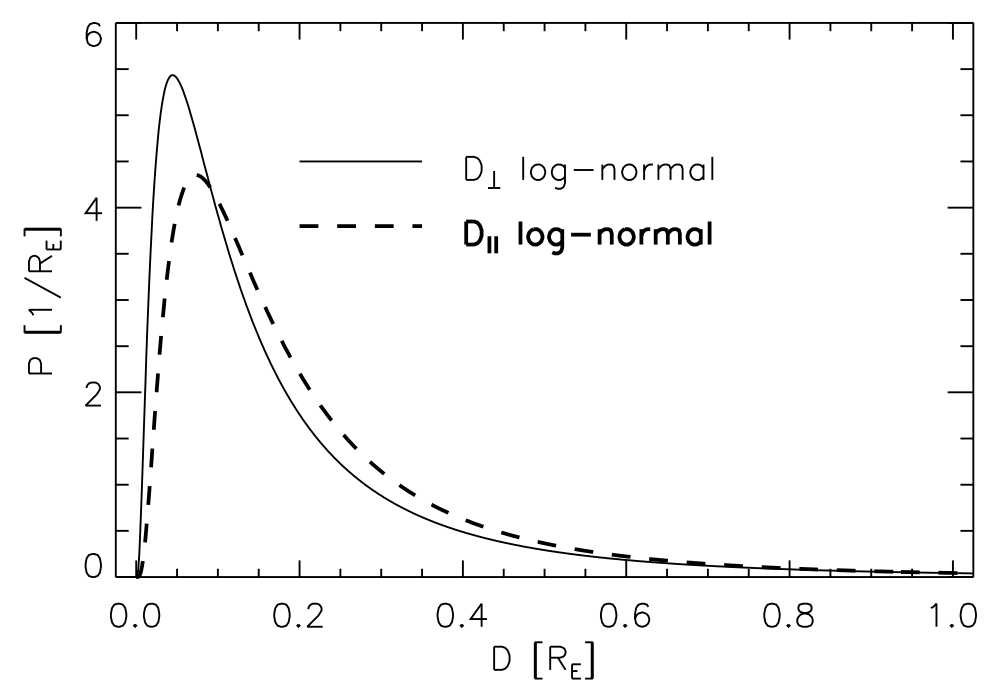

Figure 6. Unbiased jet scale size distributions $P_{\ln , \perp}$ and $P_{\ln , \|}$ (Equation 3), using parameters $\mu_{\perp}=-2.1118, \sigma_{\perp}=0.9991, \mu_{\|}=-1.8703$, and $\sigma_{\|}=0.8636$, shown by solid and dashed lines, respectively.

is also reflected in the median values $\bar{D}_{\ln }=\exp (\mu) R_{\mathrm{E}}$ of $P_{\ln , \perp}$ and $P_{\ln , \|}$, which yield $0.12 R_{\mathrm{E}}$ and $0.15 R_{\mathrm{E}}$, respectively. The values $\widehat{D}_{\mathrm{ln}}=\exp \left(\mu-\sigma^{2}\right) R_{\mathrm{E}}$ where the distributions maximize are $0.045 R_{\mathrm{E}}$ and $0.073 R_{\mathrm{E}}$, respectively. The parallel scale sizes tend to be slightly larger than the perpendicular ones, contradicting the aspect ratio inferred from $P_{\text {exp, } \perp}$ and $P_{\text {exp }, \|}$. This can also be seen in Table 2, which states the median values $\bar{D}$ of all involved distributions. The aspect ratio inversion can be traced back to the above mentioned fact that $P_{\mathrm{mod}, \perp}>P_{\mathrm{mod}, \|}$ for $D<0.1 R_{\mathrm{E}}$.

Actual jets may be even more elongated along the parallel direction, as we show by the following discussion: $\bar{D}_{\mathrm{ln}, \|}=0.15 R_{\mathrm{E}}$ has been obtained assuming a fixed jet aspect ratio. Is this assumption justified? To answer this question, we split the jet data set into two equally large groups: Group 1 contains all jets featuring interval lengths below $30 \mathrm{~s}$ (median of the jet data set); group 2 contains only those jets with jet interval lengths above $30 \mathrm{~s}$. Modeling the resultant distributions with $P_{\exp }$ (Equation 1), we obtain $D_{\| 0}=0.22 R_{\mathrm{E}}$ and $D_{\perp 0}=0.46 R_{\mathrm{E}}$ for group 1 , and $D_{\| 0}=1.48 R_{\mathrm{E}}$ and $D_{\perp 0}=$ $1.45 R_{\mathrm{E}}$ for group 2 . Temporal jet interval lengths are obviously correlated with $D_{\|}$(see Equation 11). The findings for the two separate groups show that there is also some correlation with $D_{\perp}$. However, $D_{\perp}$ appears to be less dependent on the jet interval lengths than $D_{\|}$.

Our data set is not large enough to obtain a functional form of that dependency, but we can conclude that $D_{\|}$is neither totally independent from $D_{\perp}$, nor is there a fixed aspect ratio. Consequently, the actual median parallel scale sizes should lie between those obtained under the assumption of a fixed jet aspect ratio and those obtained assuming total independence between $D_{\perp}$ and $D_{\|}$: They may be somewhat higher than $\bar{D}_{\mathrm{ln}, \|}=$ $0.15 R_{\mathrm{E}}$, but smaller than $\bar{D}_{\text {mod, } \|}=0.68 R_{\mathrm{E}}$, which is the median of the observed $D_{\|}$ distribution.

\section{Magnetopause Impact Rates}

Plaschke et al. (2016) derive impact rates of jets on the dayside magnetopause, based on the distribution $P_{\exp , \perp}$ of observed perpendicular scale sizes. They find that jets with 


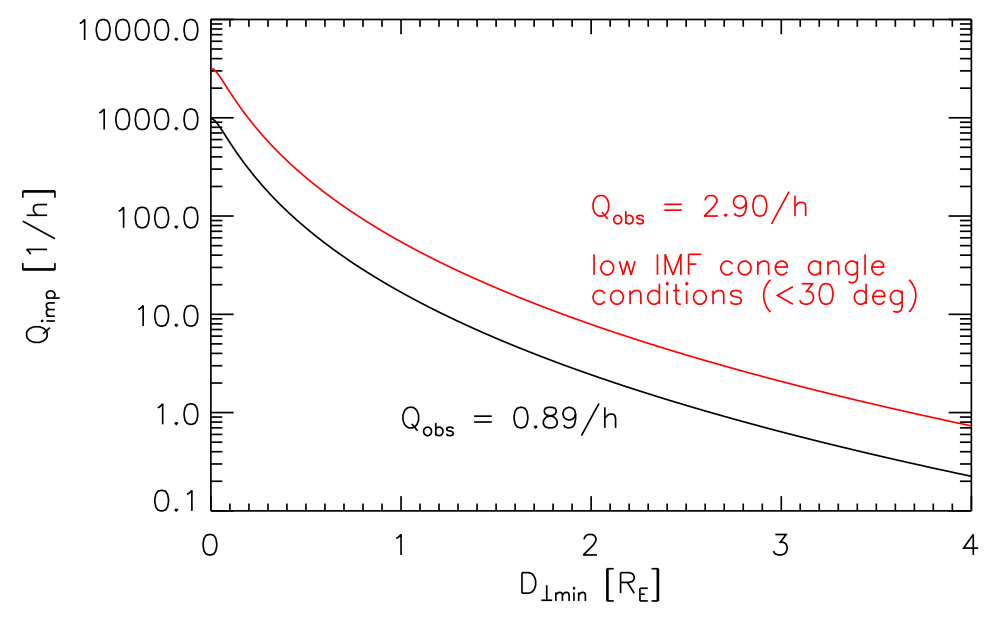

Figure 7. Jet impact rate $Q_{\text {imp }}$ (Equation 13) as a function of minimum cross-sectional diameter $D_{\perp \text { min }}$ for $Q_{\text {obs }}=0.89 / \mathrm{h}$ (black: all conditions single-spacecraft observation rate) and for $Q_{\text {obs }}=2.90 / \mathrm{h}$ (red: low IMF cone angle conditions).

cross-sectional diameters $D_{\perp}>2 R_{\mathrm{E}}$ hit the magnetopause 2.9 times per hour $(9.4 / \mathrm{h}$ for IMF cone angles below $30^{\circ}$ ). Here, $P_{\text {exp }, \perp}$ is replaced by $P_{\text {mod, }, \perp}$, raising the question of how this influences the derived impact rates. We follow the calculations outlined in section 6 of Plaschke et al. (2016) to obtain updated numbers.

The impact rate of jets of a certain minimal perpendicular (cross-sectional) size $D_{\perp \text { min }}$ on the magnetopause is given by:

$$
Q_{\text {imp }}=\int_{D_{\perp \min }}^{\infty} C P_{\text {mod }, \perp} Q_{\text {obs }} \mathrm{d} D_{\perp}
$$

Here $Q_{\text {obs }}$ is the rate of jet observation by a single spacecraft in the subsolar magnetosheath. Based on THA, THD, and THE measurements, it is $Q_{\text {obs }}=0.89 / \mathrm{h}$ in general, and $Q_{\text {obs }}=$ $2.90 / \mathrm{h}$ when the IMF cone angle does not exceed $30^{\circ} . C=A_{\text {ref }} / A_{\text {jet }}$ is the correction factor to take into account the observational bias towards larger jets. Consequently, $C \sim$ $1 / D_{\perp}^{2}$ holds. $A_{\text {ref }}=102 R_{\mathrm{E}}^{2}$ is a circular target area of the subsolar magnetopause, on which jet impacts are evaluated. $A_{\text {jet }}=\pi D_{\perp}^{2} /(4 \cos \theta)$, where $\theta=25^{\circ}$ is the mean angle between the jet propagation direction and the Earth-Sun-line, which is also normal to the target area (see Plaschke et al., 2016, for details). Therewith, Equation (12) becomes:

$$
\begin{aligned}
Q_{\text {imp }} & =\frac{4 Q_{\text {obs }} \cos \theta A_{\text {ref }} / R_{\mathrm{E}}^{2}}{\sigma_{\perp} \pi \sqrt{2 \pi} \exp \left(2 \mu_{\perp}+2 \sigma_{\perp}^{2}\right)} \int_{D_{\perp \text { min }}}^{\infty} \frac{1}{D_{\perp}} \exp \left(-\frac{\left(\ln \left(D / R_{\mathrm{E}}\right)-\mu_{\perp}\right)^{2}}{2 \sigma_{\perp}^{2}}\right) \mathrm{d} D_{\perp} \\
& =\frac{2 Q_{\text {obs }} \cos \theta A_{\text {ref }} / R_{\mathrm{E}}^{2}}{\pi \exp \left(2 \mu_{\perp}+2 \sigma_{\perp}^{2}\right)}\left[1+\operatorname{erf}\left(\frac{\mu_{\perp}-\ln \left(D_{\perp \text { min }} / R_{\mathrm{E}}\right)}{\sigma_{\perp} \sqrt{2}}\right)\right]
\end{aligned}
$$

Here erf denotes the error function. Using $Q_{\text {obs }}=0.89 / \mathrm{h}(2.90 / \mathrm{h}$ under low IMF cone angle conditions), as well as minimum cross-sectional jet diameters of $D_{\perp \min }=2 R_{\mathrm{E}}$, we obtain jet impact rates of large scale jets on the subsolar magnetopause of $2.4 / \mathrm{h}(7.9 / \mathrm{h})$. $Q_{\mathrm{imp}}$ is also shown in Figure 7 as a function of $D_{\perp \min }$.

\section{Discussion}

The values $Q_{\mathrm{imp}}=2.4 / \mathrm{h}(7.9 / \mathrm{h})$ for $Q_{\mathrm{obs}}=0.89 / \mathrm{h}(2.90 / \mathrm{h})$ can be compared with the values obtained by Plaschke et al. (2016) based on $P_{\exp , \perp}$ : They found $Q_{\mathrm{imp}}=$ 
$2.9 / \mathrm{h}(9.4 / \mathrm{h})$. Hence, the impact rates differ only by about $17 \%$, which is not surprising as $P_{\exp , \perp}$ and $P_{\text {mod }, \perp}$ are very similar for $D_{\perp}>2 R_{\mathrm{E}}$ (see solid lines in Figure 4). Consequently, all conclusions drawn in Plaschke et al. (2016) still hold, particularly those with respect to the high impact rates of large scale geoeffective jets.

Larger differences in the distributions $P_{\exp , \perp}$ and $P_{\bmod , \perp}$ are found for lower $D_{\perp}$ values, where $P_{\bmod , \perp}$ should more accurately reflect the distribution of observed scale sizes. Use of this distribution allows us to extend $Q_{\text {imp }}$ to lower minimum cross-sectional scales $D_{\perp \text { min }}$, as shown in Figure 7 . For the theoretical limit of $D_{\perp \min }=0 R_{\mathrm{E}}$, we would obtain $Q_{\text {imp }}=972 / \mathrm{h}\left(3166 / \mathrm{h}\right.$ for IMF cone angles $\left.<30^{\circ}\right)$. Hence, hundreds to thousands of jets travel towards and could potentially impact the dayside magnetopause every hour, of which the vast majority should be very small scale and probably insignificant with respect to geoeffectiveness. These small scale jets have not been accounted for in previous studies.

Note, however, that the theoretical limit of $D_{\perp \min }=0 R_{\mathrm{E}}$ is unphysical, as jets of sizes that are lower than at least one, probably a few proton gyroradii (lower end of the fluid scale) should not exist. The reason is that jets are defined by an ion fluid quantity: the ion dynamic pressure. They are, hence, intrinsically magnetohydrodynamic structures. Furthermore, an unknown fraction of the smallest scale jets may lose all their energy to the ambient plasma and die away before actually impacting the magnetopause.

Nevertheless, the question may be raised where and how jets of such small scales, on the order of $0.1 R_{\mathrm{E}}$, originate. Jets are mainly generated at the quasi-parallel bow shock. Their initial scale sizes $D_{\text {init }}$ should, hence, be related to the sizes of bow shock undulations or foreshock structures such as short large amplitude magnetic structures (SLAMS) impinging on and merging with the shock (Hietala et al., 2009, 2012; Karlsson et al., 2015, 2016). As entire "monolithic" entities, SLAMS tend to have larger sizes between $1000 \mathrm{~km}$ and $1 R_{\mathrm{E}}$ (e.g., Schwartz \& Burgess, 1991; Schwartz, 1991; Dubouloz \& Scholer, 1995; Lucek et al., 2008). However, they are also known to feature significant substructure on length scales $<600 \mathrm{~km}$, down to even 100 to $150 \mathrm{~km}$ (Lucek et al., 2004; Burgess et al., 2005).

These foreshock structures originate from wave-particle interactions in the foreshock region; they grow and steepen and interact with each other as they are convected to the shock with the solar wind flow. During this time, Equation (5) should be applicable, allowing us to close the argumentation of section 2: The sizes of the foreshock structures at the bow shock and, therewith, the jet sizes $D_{\text {init }}$ should also be log-normally distributed.

Equation (5) implies that jets evolve as they propagate in the magnetosheath. Even if they are created on scales of entire large-scale SLAMS, on the order of $1 R_{\mathrm{E}}$, it may be hypothesized that jets develop a filamentary structure or that they break up into smaller pieces before reaching the magnetopause. This may happen due to the application of viscous stresses and dynamic pressure fluctuations, and/or due to instabilities like the KelvinHelmholtz instability playing a role at interfaces between plasmas of different velocities.

In that sense, jets in the magnetosheath may undergo a multiplicative Markov process analogous to a turbulent cascade. Thereby, each break-up step should decrease the size of the jets or jet fragments by a factor proportional to $K$ in Equation (5), leading to and justifying the use of log-normal distributions to model the scale sizes of jets (e.g., Gorokhovski \& Saveliev, 2003, and references therein). Fragmentation may also explain the prevalence of jets of scale sizes on the order of $D \approx 0.1 R_{\mathrm{E}}$ near the magnetopause, as indicated by the distributions $P_{\ln , \perp}$ and $P_{\ln , \|}$. It should be noted that we can only hypothesize about the evolution of jets in the magnetosheath, since no thorough study on this issue has been conducted so far.

Note also that the underlying THEMIS observations do not cover well these particularly small scale sizes. The cadence of the ion moments used to identify the jets is 
equal to the THEMIS spacecraft spin period of approximately 3 seconds. Assuming jets to propagate with velocities on the order of $200 \mathrm{~km} / \mathrm{s}$, this time resolution would barely suffice to resolve structures on the order of $0.1 R_{\mathrm{E}}$. With respect to the perpendicular scale sizes, the minimum distance between reference and second spacecraft in any of the cases of the $2 \mathrm{SC}$ data set is $0.16 R_{\mathrm{E}}$, i.e., larger than the scales of most jets according to $P_{\mathrm{ln}, \perp}$. Consequently, there might be a relatively large error associated to $\bar{D}_{\mathrm{ln}, \perp}=0.12 R_{\mathrm{E}}$.

Furthermore, due to lacking observational capabilities with respect to small jets, it is unclear what the minimum scales of jets are. If this were known, then any observational distribution $P$ could be forced to vanish below that limit, and could also not be enhanced by any correction of observational bias. As a consequence, the median scale sizes $\bar{D}_{\text {ln }}$ as stated in Table 2 may arguably be slightly too low. Notwithstanding this, $\bar{D}_{\text {ln }}$ should be significantly smaller than $1 R_{\mathrm{E}}$ and, hence, smaller than reported in previous case and statistical studies on jets (e.g., Němeček et al., 1998; Savin et al., 2012; Hietala et al., 2012; Archer \& Horbury, 2013; Plaschke et al., 2013; Gunell et al., 2014; Plaschke et al., 2016).

Finally, we would like to note that all the quantitative results shown in this manuscript are ultimately associated to the particular set of jet identification criteria stated above in section 3. These criteria have been previously introduced and used in Plaschke et al. (2013) and Plaschke et al. (2016). Applying the same criteria again makes our results comparable to those reported in the earlier publications, as intended. However, if we defined jets differently, then we would also get different results. To date, there is no commonly accepted set of criteria to identify jets in the magnetosheath or, more generally, downstream of collisionless shocks (see also section 2 in Plaschke et al., 2018). An indepth comparison of identification criteria and resulting jet properties (e.g., sizes and impact rates) should be the subject of a future study.

\section{Summary and Conclusions}

To obtain unbiased jet scale size distributions $P_{\perp}$ and $P_{\|}$, we reanalyze jet observations following the steps described in Plaschke et al. (2016). In this previous study, exponential functions $P_{\exp }$ (Equation 1) were used as a basis for these distributions. Unfortunately, it is impossible to correct those to account for the observational bias towards larger jets: the resulting distributions cannot be normalized. Here, the exponential distributions are replaced by modified log-normal distributions $P_{\text {mod }}$ (Equation 4 ), which can be corrected and renormalized yielding log-normal distributions $P_{\ln }$ (Equation 3). We suggest that the scale sizes of jets are well-modeled by log-normal distributions, as they may be traced back to multiplicative processes in the foreshock and in the magnetosheath. This latter statement is to be confirmed in future studies, and so is our assumption of log-normally distributed sizes of bow shock undulations and/or foreshock structures that we used in the argumentation outlined in sections 2 and 8.

Based on jet observations by the THEMIS spacecraft during the years 2008 to 2011, we find the following parameters of the log-normal distributions: $\mu_{\perp}=-2.1118, \sigma_{\perp}=$ 0.9991, $\mu_{\|}=-1.8703$, and $\sigma_{\|}=0.8636$. Therewith, $P_{\ln , \perp}$ and $P_{\ln , \|}$ according to Equation (3) constitute the first estimates for unbiased distributions of jet scale sizes in the terrestrial subsolar magnetosheath. Based on these distributions, three major conclusions can be drawn.

First, jets are, in general, much smaller than reported in most, if not all, literature on this subject so far, where scale sizes on the order of $1 R_{\mathrm{E}}$ are typically stated. Median scale sizes of $\bar{D}_{\mathrm{ln}, \perp}=0.12 R_{\mathrm{E}}$ and $\bar{D}_{\mathrm{ln}, \|}=0.15 R_{\mathrm{E}}$ are found here, though $\bar{D}_{\mathrm{ln}, \|}$ arguably slightly underestimates the actual median parallel scale size, as discussed above in section 6 . The reason for the discrepancy in sizes is that the probability of jet observation scales with the jet's cross-sectional area perpendicular to the direction of prop- 
agation, i.e., with $D_{\perp}^{2}$. Hence, smaller jets are observed less frequently and are, correspondingly, heavily underrepresented in case studies and statistical analyses involving observations.

Second, $\bar{D}_{\mathrm{ln}, \perp} \approx \bar{D}_{\mathrm{ln}, \|}$ indicates that perpendicular and parallel scale sizes could be rather balanced. Factoring in that $\bar{D}_{\mathrm{ln}, \|}$ may be regarded as a lower limit, we may even conclude that jets should appear rather elongated along the parallel direction. This would be in disagreement with indications of jets being flattened along the propagation direction, based on $D_{\perp 0}=1.34 R_{\mathrm{E}} \gg D_{\| 0}=0.71 R_{\mathrm{E}}$ pertaining to the exponential observed jet scale size distributions (Plaschke et al., 2016). However, no definitive conclusion can be drawn here with respect to this matter because of lacking observations of jets of small scales, on the order of $0.1 R_{\mathrm{E}}$. Future studies using data obtained by the Cluster spacecraft in small-scale configurations (on the order of a few $100 \mathrm{~km}$ ) may shed more light on this issue.

Third, the impact rates of large scale jets, with cross-sectional diameters of $D_{\perp}>$ $2 R_{\mathrm{E}}$, on the dayside magnetopause are found to be $Q_{\mathrm{imp}}=2.4 / \mathrm{h}$ in general and $Q_{\mathrm{imp}}=$ $7.9 / \mathrm{h}$ under low IMF cone angle conditions $\left(<30^{\circ}\right)$. These values are about $17 \%$ smaller than those reported by Plaschke et al. (2016). Importantly, however, they are of the same order, i.e., the conclusions stated in that paper hold: Geoeffective jets are, indeed, very common. By deriving $Q_{\text {imp }}$ from $P_{\bmod , \perp}$, we are now able to extend the impact rate calculations to lower minimum jet scale sizes. Potentially, hundreds if not thousands of (mostly small scale) jets could impact on the magnetopause every hour. Individually, these jets are likely not (very) geoeffective. They have, however, not been accounted for at all, so far.

\section{Acknowledgments}

We acknowledge NASA contract NAS5-02099 for use of data from the THEMIS Mission, specifically C. W. Carlson and J. P. McFadden for the use of ESA data; K. H. Glassmeier, U. Auster, and W. Baumjohann for the use of FGM data provided under the lead of the Technical University of Braunschweig and with financial support through the German Ministry for Economy and Technology and the German Center for Aviation and Space (DLR) under contract 50 OC 0302. Data from the THEMIS mission including FGM and ESA data are publicly available from the University of California, Berkeley, and can be obtained from http://themis.ssl.berkeley.edu/data/themis. The OMNI solar wind data are publicly available from the NASA Space Physics Data Facility at the Goddard Space Flight Center (https://omniweb.gsfc.nasa.gov/ow_min.html). Magnetosheath and jet interval data sets are available from https://osf.io/gf732/ and the 2SC data set can be obtained from https://osf.io/vpm89/, hosted by the Open Science Framework (OSF). HH is supported by Royal Society University Research Fellowship URF $\backslash R 1 \backslash 180671$. $\mathrm{ZV}$ is supported by the Austrian FWF project P28764-N27.

\section{References}

Amata, E., Savin, S. P., Ambrosino, D., Bogdanova, Y. V., Marcucci, M. F., Romanov, S., \& Skalsky, A. (2011, May). High kinetic energy density jets in the Earth's magnetosheath: A case study. Planet. Space Sci., 59, 482-494. doi: 10.1016/j.pss.2010.07.021

Angelopoulos, V. (2008, December). The THEMIS Mission. Space Sci. Rev., 141, 534. doi: $10.1007 / \mathrm{s} 11214-008-9336-1$

Archer, M. O., Hietala, H., Hartinger, M. D., Plaschke, F., \& Angelopoulos, V. $(2019,2)$. Direct observations of a surface eigenmode of the dayside magnetopause. Nat. Commun., 10(1), 615. doi: 10.1038/s41467-018-08134-5

Archer, M. O., \& Horbury, T. S. (2013, February). Magnetosheath dynamic pressure enhancements: occurrence and typical properties. Ann. Geophys., 31, 319-331. 
doi: 10.5194/angeo-31-319-2013

Archer, M. O., Horbury, T. S., \& Eastwood, J. P. (2012, May). Magnetosheath pressure pulses: Generation downstream of the bow shock from solar wind discontinuities. J. Geophys. Res., 117, A05228. doi: 10.1029/2011JA017468

Burgess, D., Lucek, E. A., Scholer, M., Bale, S. D., Balikhin, M. A., Balogh, A., ... Walker, S. N. (2005, Jun). Quasi-parallel Shock Structure and Processes. Space Sci. Rev., 118(1-4), 205-222. doi: 10.1007/s11214-005-3832-3

Campbell, J. W. (1995, Jul). The lognormal distribution as a model for bio-optical variability in the sea. J. Geophys. Res., 100(C7), 13,237-13,254. doi: 10.1029/ 95JC00458

Dubouloz, N., \& Scholer, M. (1995, February). 2D hybrid simulations of short largeamplitude magnetic structures (SLAMS) upstream of quasi-parallel collisionless shocks. Adv. Space Res., 15, 175-178. doi: 10.1016/0273-1177(94)00100-F

Gorokhovski, M. A., \& Saveliev, V. L. (2003, January). Analyses of Kolmogorov's model of breakup and its application into Lagrangian computation of liquid sprays under air-blast atomization. Phys. Fluids, 15(1), 184-192. doi: $10.1063 / 1.1527914$

Gunell, H., Stenberg Wieser, G., Mella, M., Maggiolo, R., Nilsson, H., Darrouzet, F., ... Dandouras, I. (2014, August). Waves in high-speed plasmoids in the magnetosheath and at the magnetopause. Ann. Geophys., 32, 991-1009. doi: 10.5194/angeo-32-991-2014

Han, D.-S., Hietala, H., Chen, X.-C., Nishimura, Y., Lyons, L. R., Liu, J.-J., ... Yang, H.-G. (2017). Observational properties of dayside throat aurora and implications on the possible generation mechanisms. Journal of Geophysical Research: Space Physics, 122(2), 1853-1870. doi: 10.1002/2016JA023394

Han, D.-S., Liu, J. J., Chen, X. C., Xu, T., Li, B., Hu, Z. J., .. Pollock, C. J. (2018, Apr). Direct Evidence for Throat Aurora Being the Ionospheric Signature of Magnetopause Transient and Reflecting Localized Magnetopause Indentations. J. Geophys. Res., 123(4), 2658-2667. doi: 10.1002/2017JA024945

Han, D.-S., Xu, T., Jin, Y., Oksavik, K., Chen, X.-C., Liu, J.-J., ... Herlingshaw, K. (2019, Jul). Observational Evidence for Throat Aurora Being Associated With Magnetopause Reconnection. Geophys. Res. Lett., 46(13), 7113-7120. doi: 10.1029/2019GL083593

Hao, Y., Lembege, B., Lu, Q., \& Guo, F. (2016, March). Formation of downstream high-speed jets by a rippled nonstationary quasi-parallel shock: 2-D hybrid simulations. J. Geophys. Res., 121, 2080-2094. doi: 10.1002/2015JA021419

Hietala, H., Laitinen, T. V., Andréeová, K., Vainio, R., Vaivads, A., Palmroth, M., ... Rème, H. (2009, December). Supermagnetosonic Jets behind a Collisionless Quasiparallel Shock. Phys. Rev. Lett., 103(24), $245001 . \quad$ doi: 10.1103/PhysRevLett.103.245001

Hietala, H., Partamies, N., Laitinen, T. V., Clausen, L. B. N., Facskó, G., Vaivads, A., ... Lucek, E. A. (2012, January). Supermagnetosonic subsolar magnetosheath jets and their effects: from the solar wind to the ionospheric convection. Ann. Geophys., 30, 33-48. doi: 10.5194/angeo-30-33-2012

Hietala, H., Phan, T. D., Angelopoulos, V., Oieroset, M., Archer, M. O., Karlsson, T., \& Plaschke, F. (2018, February). In Situ Observations of a Magnetosheath High-Speed Jet Triggering Magnetopause Reconnection. Geophys. Res. Lett., 45, 1732-1740. doi: 10.1002/2017GL076525

Hietala, H., \& Plaschke, F. (2013, November). On the generation of magnetosheath high-speed jets by bow shock ripples. J. Geophys. Res., 118, 7237-7245. doi: 10.1002/2013JA019172

Karlsson, T., Kullen, A., Liljeblad, E., Brenning, N., Nilsson, H., Gunell, H., \& Hamrin, M. (2015, September). On the origin of magnetosheath plasmoids and their relation to magnetosheath jets. J. Geophys. Res., 120, 7390-7403. doi: 10.1002/2015JA021487 
Karlsson, T., Liljeblad, E., Kullen, A., Raines, J. M., Slavin, J. A., \& Sundberg, T. (2016, September). Isolated magnetic field structures in Mercury's magnetosheath as possible analogues for terrestrial magnetosheath plasmoids and jets. Planet. Space Sci., 129, 61-73. doi: 10.1016/j.pss.2016.06.002

King, J. H., \& Papitashvili, N. E. (2005, February). Solar wind spatial scales in and comparisons of hourly Wind and ACE plasma and magnetic field data. J. Geophys. Res., 110, A02104. doi: 10.1029/2004JA010649

Lucek, E. A., Horbury, T. S., Balogh, A., Dandouras, I., \& Rème, H. (2004). Cluster observations of structures at quasi-parallel bow shocks. Ann. Geophys., 22(7), 2309-2313. Retrieved from http://www. ann-geophys.net/22/2309/2004/ doi: 10.5194/angeo-22-2309-2004

Lucek, E. A., Horbury, T. S., Dandouras, I., \& Rème, H. (2008). Cluster observations of the earth's quasi-parallel bow shock. J. Geophys. Res., 113(A7), n/an/a. Retrieved from http://dx.doi.org/10.1029/2007JA012756 (A07S02) doi: 10.1029/2007JA012756

McFadden, J. P., Carlson, C. W., Larson, D., Ludlam, M., Abiad, R., Elliott, B., ... Angelopoulos, V. (2008, December). The THEMIS ESA Plasma Instrument and In-flight Calibration. Space Sci. Rev., 141, 277-302. doi: 10.1007/s11214-008-9440-2

Němeček, Z., Šafránková, J., Přech, L., Sibeck, D. G., Kokubun, S., \& Mukai, T. (1998). Transient flux enhancements in the magnetosheath. Geophys. Res. Lett., 25, 1273-1276. doi: 10.1029/98GL50873

Palmroth, M., Hietala, H., Plaschke, F., Archer, M., Karlsson, T., Blanco-Cano, X., ... Turc, L. (2018, September). Magnetosheath jet properties and evolution as determined by a global hybrid-Vlasov simulation. Ann. Geophys., 36, 1171-1182. doi: 10.5194/angeo-36-1171-2018

Plaschke, F., \& Glassmeier, K.-H. (2011, October). Properties of standing KruskalSchwarzschild-modes at the magnetopause. Ann. Geophys., 29, 1793-1807. doi: 10.5194/angeo-29-1793-2011

Plaschke, F., Hietala, H., \& Angelopoulos, V. (2013, October). Anti-sunward highspeed jets in the subsolar magnetosheath. Ann. Geophys., 31, 1877-1889. doi: 10.5194/angeo-31-1877-2013

Plaschke, F., Hietala, H., \& Angelopoulos, V. (2020, Aug). Anti-sunward high-speed jets in the subsolar magnetosheath: Data sets. OSF. Retrieved from osf.io/ gf732

Plaschke, F., Hietala, H., Angelopoulos, V., \& Nakamura, R. (2016). Geoeffective jets impacting the magnetopause are very common. J. Geophys. Res., 121, 3240-3253. doi: 10.1002/2016JA022534

Plaschke, F., Hietala, H., Angelopoulos, V., \& Nakamura, R. (2020, Aug). Geoeffective jets impacting the magnetopause are very common: Data set. OSF. Retrieved from osf.io/vpm89

Plaschke, F., Hietala, H., Archer, M., Blanco-Cano, X., Kajdič, P., Karlsson, T., ... Sibeck, D. (2018, August). Jets Downstream of Collisionless Shocks. Space Sci. Rev., 214, 81. doi: 10.1007/s11214-018-0516-3

Plaschke, F., Karlsson, T., Hietala, H., Archer, M., Vörös, Z., Nakamura, R., ... Giles, B. L. (2017). Magnetosheath high-speed jets: Internal structure and interaction with ambient plasma. J. Geophys. Res., 122(10), 10,157-10,175. doi: 10.1002/2017JA024471

Savin, S., Amata, E., Zelenyi, L., Nemecek, Z., Borodkova, N., Buechner, J., ... Lezhen, L. (2012). Super fast plasma streams as drivers of transient and anomalous magnetospheric dynamics. Ann. Geophys., 30, 1-7. doi: doi:10.5194/angeo-30-1-2012

Schwartz, S. J. (1991). Magnetic field structures and related phenomena at quasiparallel shocks. Adv. Space Res., 11, 231-240. doi: 10.1016/0273-1177(91)90039 $-\mathrm{M}$ 
Schwartz, S. J., \& Burgess, D. (1991, March). Quasi-parallel shocks - A patchwork of three-dimensional structures. Geophys. Res. Lett., 18, 373-376. doi: 10 $.1029 / 91$ GL00138

Shue, J.-H., Chao, J.-K., Song, P., McFadden, J. P., Suvorova, A., Angelopoulos, V., ... Plaschke, F. (2009, September). Anomalous magnetosheath flows and distorted subsolar magnetopause for radial interplanetary magnetic fields. Geophys. Res. Lett., 36, L18112. doi: 10.1029/2009GL039842

Vörös, Z., Leitner, M., Narita, Y., Consolini, G., Kovács, P., Tóth, A., \& Lichtenberger, J. (2015, Aug). Probability density functions for the variable solar wind near the solar cycle minimum. J. Geophys. Res., 120(8), 6152-6166. doi: 10.1002/2015JA021257

Vuorinen, L., Hietala, H., \& Plaschke, F. (2019). Jets in the magnetosheath: Imf control of where they occur. Ann. Geophys., 37, 689-697.

Wang, B., Nishimura, Y., Hietala, H., Lyons, L., Angelopoulos, V., Plaschke, F., ... Weatherwax, A. (2018, Jun). Impacts of Magnetosheath High-Speed Jets on the Magnetosphere and Ionosphere Measured by Optical Imaging and Satellite Observations. J. Geophys. Res., 123(6), 4879-4894. doi: 10.1029/2017JA024954 